\title{
Budgetary Impact of Treatment with Adjuvant Imatinib for 1 Year Following Surgical Resection of Kit-Positive Localized Gastrointestinal Stromal Tumors
}

\author{
Jaime L. Rubin, MA; Douglas C.A. Taylor, MBA; Myrlene Sanon, MPH; \\ John H. Coombs, PharmD, MBA; and Vamsi K. Bollu, PhD, MBA
}

\begin{abstract}
BACKGROUND: Imatinib mesylate, an orally administered kinase inhibitor that targets the KIT (CD117) protein, currently has 10 approved indications including treatment of chronic myelogenous leukemia and metastatic gastrointestinal stromal tumors (GIST). Treatment with adjuvant imatinib following surgical resection of localized Kit-positive GIST, the most recent FDA-approved indication (December 2008), has been shown to significantly improve recurrence-free survival (RFS) compared with surgical resection alone. Although adjuvant imatinib has proven effective in clinical trials, it is important to consider the economic impact to health plans of introducing imatinib in accordance with its new labeled indication.
\end{abstract}

OBJECTIVE: To evaluate the budgetary impact over a 3-year time horizon of treating patients with localized Kit-positive GIST with 1 year of adjuvant imatinib following surgical resection.

METHODS: A Markov model was developed to predict patients' transitions across health states defined by initial treatment (surgical resection followed by adjuvant imatinib $\mathbf{4 0 0}$ milligrams [mg] daily versus surgical resection alone), recurrence, and progression. Treatments for a first recurrence were (a) imatinib $400 \mathrm{mg}$ daily for recurrences following resection only or after completion of 1 year of treatment with imatinib $400 \mathrm{mg}$ daily and (b) imatinib $800 \mathrm{mg}$ daily for recurrence during active treatment with imatinib $400 \mathrm{mg}$ daily. Treatments for further progression were imatinib $800 \mathrm{mg}$ daily, sunitinib, or best supportive care (BSC) following imatinib $400 \mathrm{mg}$ per day, and sunitinib or BSC following imatinib $800 \mathrm{mg}$ daily. Recurrence rates were derived from the American College of Surgeons Oncology Group (ACOSOG) Z9001 clinical trial, which compared 1 year of adjuvant imatinib following surgical resection with surgical resection only. The total number of patients with localized and surgically resected GIST (incidence rate of 0.36 per 100,000 ) was estimated from epidemiologic studies of GIST. Uptake of treatment with imatinib was estimated from unpublished data from qualitative market research funded by the study sponsor. The uptake rate assumptions reflected both (a) the percentage of patients with Kitpositive disease and (b) the percentage of clinically eligible patients who would use imatinib. Costs were estimated by combining unit costs from published sources with expected resource utilization based on the clinical trial publication and National Comprehensive Cancer Network guidelines on the treatment of patients with GIST. To obtain estimates of the budgetary impact, we compared estimated health care costs with versus without adjuvant imatinib, where health care costs with imatinib reflected the costs of treatment minus cost offsets associated with delayed or avoided recurrence or progression. All "with" scenarios assumed no additional uses other than surgically resected localized Kit-positive GIST (i.e., no change in off-label use of imatinib). The budgetary impact was estimated for the first 3 years after the introduction of adjuvant imatinib in accordance with its new labeled indication in a hypothetical plan population of 10 million persons. Results were calculated both as total budgetary impact and as per member per month (PMPM) cost in 2009 dollars. Sensitivity analyses were performed to test the robustness of model results to changes in parameter estimates.
RESULTS: The model predicted 36 incident resected GIST cases per year in a health plan of 10 million members. The estimated counts of cases treated with adjuvant imatinib were 10.8, 16.2, and 21.6 in the first, second, and third years after introduction, respectively, with the annual increases attributable to changes in the proportion of patients with resected GIST assumed to use imatinib (30\% in year 1, rising to $45 \%$ in year 2 and $60 \%$ in year 3 ). The model predicted that treatment of these cases with imatinib will increase pharmacy costs by an additional $\$ 505,144$ in the first year, $\$ 757,717$ in the second year, and $\$ 1,010,289$ in the third year. Increased resource use associated with monitoring patients during and after treatment with adjuvant imatinib would cost an additional $\$ 21,564, \$ 38,145$, and $\$ 56,605$ in the first, second, and third years, respectively. Recurrence would be avoided or delayed in 7 patients over the 3 -year period. Avoided or delayed recurrences would result in cost offsets of $\$ 61,583$ in the first year, $\$ 156,702$ in the second year, and $\$ 233,849$ in the third year. The net budgetary impact was estimated to be $\$ 465,126$ in the first year (less than \$0.01 PMPM), \$639,159 in the second year (\$0.01 PMPM), and \$833,044 in the third year (\$0.01 PMPM). Results of sensitivity analyses indicated that the budgetary impact in the third year is most sensitive to changes in the price of adjuvant imatinib and recurrence rates.

CONCLUSIONS: The model predicted that the introduction of adjuvant imatinib for treatment of surgically resected, localized, Kit-positive GIST will lead to a net budgetary impact of \$0.01 PMPM in the third year after introduction assuming change in use only in accordance with the new labeled indication. Approximately $11.7 \%-21.9 \%$ of the cost of adjuvant imatinib is offset by the reduction in costs associated with GIST recurrence.

\section{J Manag Care Pharm. 2010;16(7):482-91}

Copyright $\odot 2010$, Academy of Managed Care Pharmacy. All rights reserved.

\section{What is already known about this subject}

- Gastrointestinal stromal tumor (GIST) is a sarcoma of the gastrointestinal tract that affects approximately 3,000-4,000 individuals per year in the United States, or about 1 to 2 patients per 100,000 health plan members.

- Localized disease accounts for approximately 30\%-50\% of all GIST cases, is associated with high risk of recurrence (more than $40 \%$ in first 2 years after surgical resection), and is treated with surgery and sometimes adjuvant imatinib. Imatinib was approved in the United States as adjuvant therapy in patients with surgically resected Kit-positive GIST in December 2008.

- In the American College of Surgeons Oncology Group (ACOSOG) Z9001 study, a randomized, double-blind, placebo-controlled trial, treatment with adjuvant imatinib following surgical resection of localized Kit-positive GIST significantly improved recurrence-free survival compared with surgical resection alone $(98 \%$ vs. $83 \%$, respectively, hazard ratio $[\mathrm{HR}]=0.35,95 \% \mathrm{CI}=0.22$ 0.53 , at 1 year) 


\section{What this study adds}

- We developed a decision analytic model to estimate the projected budgetary impact of treating localized GIST patients with 1 year of adjuvant imatinib following surgical resection in a health plan with 10 million members. Assuming annual incidence of 0.36 per 100,000 members (i.e., 36 patients with localized resected GIST each year) and increasing uptake over time, the predicted counts of incident cases would be 10.8 in the first year, 16.2 in the second year, and 21.6 in the third year.

- The model predicted that treatment with adjuvant imatinib will cost an additional $\$ 526,709$ in the first year, $\$ 795,861$ in the second year, and $\$ 1,066,893$ in the third year, inclusive of pharmacy costs and additional resource use associated with monitoring patients on adjuvant imatinib. Cost offsets associated with delayed and avoided GIST recurrences were predicted at $\$ 61,583$ in the first year, $\$ 156,702$ in the second year, and $\$ 233,849$ in the third year. After accounting for cost offsets, the predicted net costs of adjuvant imatinib in the first, second, and third years were $\$ 465,126, \$ 639,159$, and $\$ 833,044$, respectively. The predicted budgetary impact was less than \$0.01 PMPM in the first year and about $\$ 0.01$ in the second and third years.

- When disease incidence was increased to twice the estimated base-case incidence $(0.72$ per 100,000$)$ in sensitivity analyses, the budgetary impact of adjuvant imatinib in a health plan with 10 million members ranged from $\$ 929,204$ in the first year to $\$ 1.664$ million in the third year, and the annual PMPM was approximately the same as in the base-case analysis. The budgetary impact was most sensitive to changes in drug costs and the risk of recurrence.

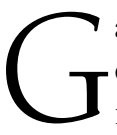
astrointestinal stromal tumors (GISTs) are mesenchymal tumors of the intestinal tract occurring most frequently in the stomach and small intestine and less frequently in the colon, rectum, and esophagus. ${ }^{1}$ GISTs are rarely found outside the gastrointestinal tract. ${ }^{2}$ It is estimated that GIST, the most common type of sarcoma in the intestinal tract, affects about 3,000-4,000 persons each year in the United States. ${ }^{3-5}$ Recent research has confirmed that GISTs are often the result of mutations of the c-KIT receptor. ${ }^{6,7}$ The identification of these mutations has led to advances in targeted therapies for the treatment of GIST.7-9 Imatinib (Gleevec/Glivec; Novartis), a tyrosine kinase inhibitor (TKI) that targets the KIT protein, is currently approved by the U.S. Food and Drug Administration (FDA) for 10 indications, including treatment of unresectable and/or metastatic GIST and as adjuvant treatment of adult patients following surgical resection. ${ }^{10}$

Resectable localized disease was historically treated with surgical resection and conventional chemotherapy ${ }^{11}$ however, prior to the availability of TKI therapy, chemotherapy did not appear to prolong survival in GIST patients. ${ }^{7,11}$ Until recently, resectable localized disease was often treated with complete surgical resection only ${ }^{5}$ without adjuvant pharmacotherapy because effective therapies were not available. Although surgical resection is the recommended treatment for localized disease, ${ }^{12}$ recurrence following resection is common, with greater than $40 \%$ of patients experiencing a recurrence within the first 2 years after surgery. ${ }^{7}$ Five-year survival among patients with localized disease treated with resection only is $54 \%{ }^{7}$

The American College of Surgeons Oncology Group (ACOSOG) Z9001 study, a randomized, double-blind, placebocontrolled trial, showed that treatment with adjuvant imatinib for 1 year significantly improved recurrence-free survival (RFS) in patients with surgically resected Kit-positive GIST compared with surgical resection alone (1-year RFS was 98\% for adjuvant imatinib, $83 \%$ for placebo). ${ }^{1}$ The clinical trial was terminated early (median follow-up of 14 months) due to significant RFS benefits associated with imatinib; the final published study results represented a median follow-up time of 19.7 months. The FDA approved the use of imatinib as adjuvant therapy in surgically resected Kit (CD117) positive GIST patients in December 2008, based on the 14-month results of the ACOSOG Z9001 study. ${ }^{13}$ At a median follow-up of 14 months, the 1-year hazard ratio (HR) for recurrence or death was 0.40 (95\% confidence interval $[\mathrm{CI}]=0.26-0.61$ ) for imatinib versus surgical resection only. At 19.7 months, the corresponding 1-year HR was 0.35 (95\% CI=0.22-0.53). ${ }^{1,13}$

Although adjuvant imatinib has the potential to improve RFS in patients with surgically resected GIST, it is important to assess the impact to payers of introducing newly approved therapies. We therefore used techniques of decision analysis to model the potential budgetary impact to a hypothetical U.S. managed care plan of the use of adjuvant imatinib in accordance with its most recent labeled indication, use in patients with Kit-positive surgically resected GIST.

\section{Methods}

\section{Model Overview}

Budgetary impact analysis assesses the impact of adopting a novel intervention on health plan costs by comparing a current scenario, which does not include the novel therapy, with a future scenario, which does include the novel therapy. We constructed a decision analytic model to assess the budgetary impact of treating surgically resected Kit-positive GIST patients with 1 year of adjuvant imatinib over a 3-year time horizon. Specifically, estimated health care costs were compared with versus without imatinib in the adjuvant GIST setting. Overall budgetary impact was calculated as net cost, reflecting the costs of treatment with adjuvant imatinib and any other associated costs (e.g., monitoring costs) minus cost offsets associated with delayed recurrence or slowed disease progression.

There are 3 main components of the budgetary impact model: the population component, the marketplace dynamics component, and the economic component. The population component defines the total number of patients eligible for treatment with adjuvant imatinib in accordance with the new labeled indication. The marketplace dynamics component 


\section{FIGURE 1 Markov Model Tree Structure Diagram}

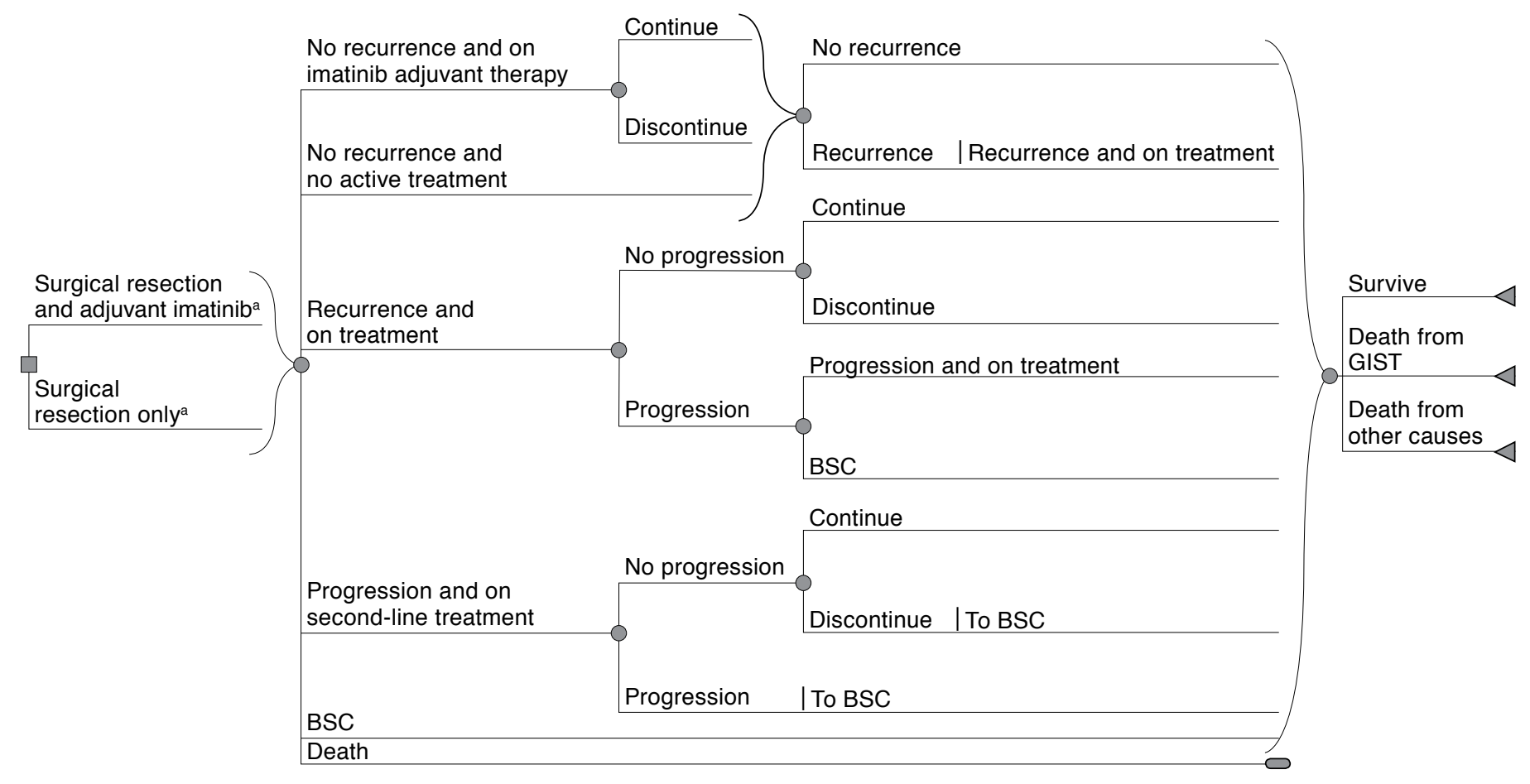

aAll patients start in the "no recurrence and no active treatment" state or the "no recurrence and on imatinib adjuvant therapy" state depending on treatment choice. BSC = best supportive care; GIST = gastrointestinal stromal tumors.

models the market share and uptake of adjuvant imatinib after its introduction to the market. The economic component predicts costs and cost offsets that result from the introduction of imatinib in the adjuvant setting.

\section{Model Structure and Estimation}

Techniques of decision analysis were used to construct and estimate a Markov model of treatment, outcomes, and costs for patients who undergo complete gross resection of primary localized GIST. The model focused on disease recurrence and progression as predictors of costs and outcomes following surgical resection.

The model structure was based on the ACOSOG Z90001 clinical trial protocol, National Comprehensive Cancer Network (NCCN) and European Society for Medical Oncology (ESMO) guidelines for the treatment of patients with GIST, and expert opinion..$^{1,12,14}$ The model was structured in the form of a Markov process, with the primary health states grouping patients into the following 6 categories: (a) free of recurrent GIST and treated with adjuvant imatinib; (b) free of recurrent GIST and no active treatment; (c) recurrent GIST and on active treatment; (d) progression and on active second-line treatment; (e) best supportive care (BSC); or (f) death (Figure 1). All patients were assumed to begin the model free of recurrent GIST and to be either treated with adjuvant imatinib or receive no additional treatment following surgical resection. In subsequent cycles of the Markov process, some patients remained in their originally assumed state, while others transitioned to the recurrent GIST or death states, based on probabilities associated with the treatment received (i.e., resection only vs. resection plus adjuvant treatment with imatinib). One year of treatment was used in the model in accordance with the ACOSOG Z9001 trial data used to obtain the FDA approval. The Markov model assumed that transitions between health states occur at 1-month intervals (1-month cycle length). This process continued until the time horizon of 3 years was reached (36 1-month cycles). All patients were assumed to begin the model at age 58 years to reflect the mean age of patients in the ACOSOG Z9001 trial. ${ }^{1}$

Patients receiving adjuvant therapy were assumed to be subject to treatment-related adverse events (AEs) and subsequent discontinuation of treatment. Discontinuation due to AEs incurred costs.

It was assumed that during each model cycle (a) patients who do not have recurrent GIST are at risk of recurrence, (b) patients who experience a recurrence are subsequently subject to a disease progression based on treatment-specific rates, and 
(c) patients who do not experience progression continue treatment. In accordance with the ACOSOG Z9001 study protocol, (a) treatments for a first recurrence were assumed to be imatinib $400 \mathrm{mg}$ daily following resection only and imatinib 800 mg daily for patients who experience a first recurrence during active treatment with imatinib $400 \mathrm{mg}$ daily, and (b) patients who experience a first recurrence after completion of 1 year of adjuvant treatment with imatinib $400 \mathrm{mg}$ daily were assumed to be retreated with imatinib $400 \mathrm{mg}$ daily. ${ }^{1}$

Patients who progress while treated for first recurrence were assumed to receive either second-line therapy or BSC. Specifically, patients were assumed to be eligible for treatment with an increased dose of imatinib (800 mg per day second-line treatment), sunitinib (Sutent; Pfizer), or receive BSC if their first recurrence was treated with imatinib $400 \mathrm{mg}$ per day. Patients whose first recurrence was treated with imatinib $800 \mathrm{mg}$ per day were assumed to be eligible for treatment with sunitinib or receive BSC. Patients who discontinue therapy because imatinib for recurrence is not well tolerated were assumed either to be treated with sunitinib or to receive BSC. All patients who fail on second-line treatment (i.e., progress while taking either imatinib $800 \mathrm{mg}$ per day or sunitinib second-line treatment) were assumed to transition to BSC; once in BSC, patients were assumed to remain there until death.

Data from published literature and the advice of 4 physicians with expertise in the treatment of GIST patients were used to estimate model input parameters (Tables 1 and 2).

\section{Population Module}

Because the budgetary impact is largely dependent on the number of patients receiving adjuvant imatinib, the determination of how many patients are candidates for adjuvant therapy constitutes an important focus of the budgetary impact analysis. The size of the eligible patient population for adjuvant imatinib was estimated from an analysis of the Surveillance, Epidemiology, and End Results (SEER) database using SEER*stat. ${ }^{15,16}$ Patients were identified using indications of (a) a GIST diagnosis in the SEER database in either 2001 or 2002; (b) localized disease as defined by the SEER histologic stage variable; and (c) surgical resection, identified by the SEER variable indicating surgical resection of the primary tumor. Assessment of whether the GIST was Kit-positive could not be made using the SEER database and was instead incorporated into market uptake assumptions. Diagnosis of GIST was based on either the International Classification of Diseases for Oncology (ICD-O)-3 for GIST (8936/3) or the algorithm validated by Tran et al. (2005). ${ }^{3}$ The Tran algorithm consists of ICD-O-2 codes for mesenchymal tumors $(8680,8711,8800-8804,8810-8811$, 8890-8893, 8896-8897, 8930, 8990, 9120, 9150, 9490, 9491, $9500,9503,9505,9540,9560)$ combined with codes for the primary sites typical for GIST: esophagus (150-159); stomach (160-169); small intestine (170-179); colon and rectum (180-
189, 199, 260, 299); retroperitoneum (480); and peritoneum, omentum, mesentery (481-482).

The annual incidence of surgically resected localized GIST was estimated as an average over the 2-year period (2001-2002) to be 0.36 per 100,000 population in the SEER analysis. This incidence rate was used to estimate the size of the patient cohort entering the model each year. The budgetary impact was assessed for a hypothetical plan population of 10 million persons.

\section{Marketplace Dynamics Module}

The expected proportion of eligible patients treated with adjuvant imatinib and the annual uptake of adjuvant imatinib after its introduction were estimated based on proprietary, unpublished data from qualitative market research funded by the study sponsor. The uptake rate assumptions reflected both (a) the percentage of patients with Kit-positive disease, estimated at $75 \%$ of patients with surgically resected localized GIST; and (b) the percentage of clinically eligible patients who would use imatinib. It was estimated that in the first year after introduction, 30\% of patients with localized GIST would receive adjuvant imatinib following surgical resection; market uptake was assumed to increase to $45 \%$ in the second year and $60 \%$ in the third year. All localized resected GIST patients not receiving adjuvant imatinib were assumed to receive no pharmacotherapy initially after surgical resection. The analysis considers only patients with Kit-positive localized GIST being treated with adjuvant imatinib after surgical resection; the numbers of patients treated with adjuvant imatinib based on market shares assumed no new off-label use of the product.

\section{Economic Module}

Probabilities of Recurrence. Monthly probabilities of first recurrence were derived from the clinical trial publication of the ACOSOG Z9001 study. ${ }^{1}$ Data were presented for up to 48 months of follow-up in the clinical trial publication. We therefore used trial data directly to estimate recurrence probabilities for each of the 3 years of the model for both the imatinib and surgical resection only arms. RFS probabilities from the trial report were converted to probabilities of recurrence by first subtracting reported overall survival from RFS and then subtracting the proportion recurrence-free (RF) from 1. Annual probabilities were converted to monthly probabilities as $1-\mathrm{RF}^{(1 / 12)}$.

Discontinuation. Persons treated with adjuvant imatinib in the model were assumed to discontinue treatment at rates reported in the publication of the clinical trial. Because the trial data were reported from an intent-to-treat perspective, discontinuation observed in the trial is already reflected in the recurrence rates reported. We assumed that patients discontinued only due to adverse events (i.e., 16\% discontinued during a 


\section{Budgetary Impact of Treatment with Adjuvant Imatinib for 1 Year Following Surgical Resection of Kit-Positive Localized Gastrointestinal Stromal Tumors}

\section{TABLE 1 Probabilities of Recurrence, Discontinuation, and Death, and Treatment Pathways}

Parameter Probabilities of Recurrence, Discontinuation, and Deat Source

Monthly probability of recurrence for patients with recurrence-free GIST

\begin{tabular}{l|l}
\hline Adjuvant imatinib $400 \mathrm{mg}$ daily & 0.0019 \\
\hline First year (on treatment) & 0.0075 \\
\hline Second year (off treatment) & 0.0102 \\
\hline Third year (off treatment) & 0.0151 \\
\hline Surgical resection only & 0.0093 \\
\hline First year after surgical resection & 0.0016 \\
\hline Second year after surgical resection & \\
\hline Third year after surgical resection &
\end{tabular}

Treatment for recurrence/progression during or after adjuvant imatinib (\%)

First recurrence while on active treatment with imatinib $400 \mathrm{mg}$ per day:

imatinib $800 \mathrm{mg}$ per day

First recurrence after completing l year of adjuvant imatinib $400 \mathrm{mg}$ per day:

retreatment with imatinib $400 \mathrm{mg}$ per day

Progression

Sunitinib second-line treatment

Best supportive care

Treatment for recurrence/progression following surgical resection only (\%)

First recurrence: imatinib $400 \mathrm{mg}$ per day

Progression

Imatinib $800 \mathrm{mg}$ per day second-line treatment

Sunitinib second-line treatment

Best supportive care

Monthly probability of progression while treated with

Imatinib $400 \mathrm{mg}$ per day for first recurrence

Imatinib $800 \mathrm{mg}$ per day for first recurrence

Imatinib $800 \mathrm{mg}$ per day second-line therapy

Sunitinib second-line therapy

Monthly probability of discontinuation

Adjuvant imatinib (due to adverse events)

Imatinib $400 \mathrm{mg}$ per day first-line treatment for recurrence

Imatinib $800 \mathrm{mg}$ per day first-line treatment for recurrence

100

100

DeMatteo et al., 20091,a

Monthly probability of death from GIST following GIST recurrence

Imatinib $400 \mathrm{mg}$ per day first-line treatment

Imatinib $800 \mathrm{mg}$ per day first-line treatment

Imatinib $800 \mathrm{mg}$ per day second-line treatment

Sunitinib second-line treatment

Best supportive care

Monthly probability of death from other causes

Age 58 years

Age 59 years

Age 60 years

aTo estimate the monthly probability of recurrence, annual probability of recurrence was derived from RFS in Figure 2 in DeMatteo et al. 2009.1 We removed mortality from RFS (based on OS in Figure 4 in DeMatteo et al.) to arrive at the probability of no recurrence (p) and estimated the monthly probability of recurrence as $1-p^{(1 / 12)}$. Estimated RFS (OS) for imatinib was $0.975(0.997)$ in year 1, 0.884 (0.990) in year 2, and $0.755(0.965)$ in year 3. Estimated RFS (OS) for surgical resection only was 0.830 (0.997) in year 1, $0.723(0.979)$ in year 2, and $0.730(0.956)$ in year 3. Because patients in the trial were treated for only 1 year, trial data from years 2 and 3 represent RFS for patients after they stopped active treatment with adjuvant imatinib.

bexpert assessment: 4 U.S. GIST opinion leaders were surveyed and asked to describe treatment patterns for their patients with GIST. Best supportive care indicates no active treatment 'Estimated using 2-year PFS reported in Verweij et al. 20048 for the imatinib $400 \mathrm{mg}$ per day arm; 2-year PFS was estimated as 0.44 and converted to a monthly probability of recurrence as $1-P F S^{(1 / 24)}$.

Estimated using 2-year PFS reported in Verweij et al. 20048 for the imatinib $400 \mathrm{mg}$ BID arm (0.50); 2-year PFS was converted to a monthly rate of recurrence as 1-PFS(1/24). eEstimates include both recurrence and discontinuation due to AEs. Risk of recurrence was estimated from the median PFS in Contreras-Hernández et al. $20088^{18}$ (5.28 months) and the risk of discontinuation due to an AE reported in Verweij et al. $2004^{8}$ (7\% over 25 months). Annual probabilities were converted to monthly probabilities as $1-P F S^{(1 / 24)}$

${ }^{f}$ Estimates include both recurrence and discontinuation due to AEs. Adapted from the median time to progression reported for the ITT sunitinib arm (27.3 weeks) in Demetri et al. 2006,17 converted to monthly probability of recurrence as 1-0.5 (1/6.3) and the risk of discontinuation due to an AE (9\%) reported in Demetri et al. $2006 .{ }^{17}$

gEstimated based on Figure 1 in DeMatteo et al. 2009:1 16\% discontinued due to AEs. Monthly probability of discontinuation estimated as 1-(1-discontinuation) (1/12)

hBased on $7 \%$ discontinuation in approximately 25 months reported in Verweij et al. 2004:8 estimated as 1-(1-0.07)(1/25)

iAdapted from Verweij et al. 2004:8 estimated from reported 1-year OS of 85\% for imatinib $400 \mathrm{mg}$ per day and 86\% for imatinib $400 \mathrm{mg}$ BID (e.g., 1-0.85(1/12)).

jAdapted from Demetri et al. 2006;17 estimated from reported median OS of 73 weeks. It was assumed that persons on second-line treatment with $800 \mathrm{mg}$ per day imatinib

had the same OS as those on sunitinib second-line treatment. Estimated as 1-0.5(1/16.8)

${ }^{k}$ Estimated as the average OS reported by Huse et al. 200720 (median OS of 20 months), Tran et al. 20053 (1-year OS of 77\%), and Demetri et al. $2006^{17}$ (median OS of 36 weeks) ${ }^{l}$ Estimated directly from U.S. life tables 2004,19 where annual probabilities were converted to monthly probabilities.

$A E=$ adverse event; $B I D=$ twice daily; $G I S T=$ gastrointestinal stromal tumors; $I T T=$ intention-to-treat; $m g=$ milligrams; OS=overall survival; PFS=progression-free survival; $R F S=$ recurrence-free survival. 


\section{TABLE 2 Monthly Costs Associated with GIST and GIST Recurrence}

\begin{tabular}{|c|c|c|}
\hline Parameter & Value & Source/Comment \\
\hline \multicolumn{3}{|l|}{ Resource costs ${ }^{24}$} \\
\hline Gastroenterologist visit & $\$ 99$ & CPT codes 99213,99215 \\
\hline CT scan & $\$ 331$ & CPT codes $74150,74160,74170$ \\
\hline General practitioner visit & $\$ 39$ & CPT code 99212 \\
\hline \multicolumn{3}{|l|}{ Monthly pharmacy costs ${ }^{23}$} \\
\hline Imatinib $400 \mathrm{mg}$ & $\$ 4,340$ & Based on 30-day supply of imatinib $400 \mathrm{mg}$ per day. \\
\hline Imatinib $800 \mathrm{mg}$ & $\$ 8,681$ & Assumes that imatinib $800 \mathrm{mg}$ per day is twice the price of $400 \mathrm{mg}$ per day. \\
\hline Sunitinib & $\$ 6,177$ & $\begin{array}{l}\text { Assumes } 4 \text { weeks on treatment with sunitinib } 50 \mathrm{mg} \text { per day and } 2 \text { weeks off treatment, averaged } \\
\text { over a l-year period and divided by } 12 \text {. }\end{array}$ \\
\hline \multicolumn{3}{|c|}{ Monthly monitoring and medical costs by health state } \\
\hline \multicolumn{3}{|c|}{ Recurrence-free GIST } \\
\hline No treatment & $\$ 120$ & $\begin{array}{l}\text { Because high-risk (defined as tumors large in size, with high mitotic count, and c-KIT exon } 11 \\
\text { mutation) and low-risk patients will have different resource use, we estimated resource use and } \\
\text { costs for high- and low-risk patients separately and calculated a weighted average cost for high- } \\
\text { and low-risk patients using the proportion in each stratum in the Z9001 study }{ }^{1} \text { and ESMO treat- } \\
\text { ment guidelines }{ }^{12} \text { to determine service use. Low-risk: } 1 \text { GE per } 6 \text { months, } 1 \text { CT per } 6 \text { months, } 2 \\
\text { GP per year. High-risk: } 1 \text { GE per } 3 \text { months, } 1 \text { CT per } 3 \text { months, } 2 \text { GP per year. }{ }^{a}\end{array}$ \\
\hline \multicolumn{3}{|r|}{ 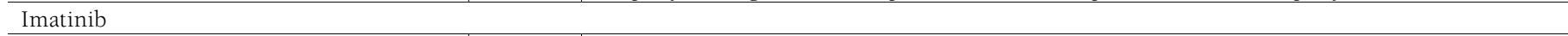 } \\
\hline First 3 months & $\$ 522$ & $\begin{array}{l}\text { Frequency of visits/tests based on trial protocol from the Z9001 study. }{ }^{1} \text { In the first } 3 \text { months, } 5 \\
\text { GE, } 3 \text { CT; } 2 \text { GP per year. }\end{array}$ \\
\hline 4 months to 2 years & $\$ 150$ & $\begin{array}{l}\text { Frequency of visits/tests based on trial protocol from the Z9001 study. }{ }^{1} 1 \text { GE every } 3 \text { months, } 1 \\
\text { CT every } 3 \text { months, } 2 \text { GP per year. Costs were applied to all patients in the adjuvant imatinib arm } \\
\text { who have not experienced a recurrence. }\end{array}$ \\
\hline Year 3 & $\$ 78$ & $\begin{array}{l}\text { Frequency of visits/tests based on trial protocol from the Z9001 study. }{ }^{1} 1 \text { GE every } 6 \text { months, } 1 \\
\text { CT every } 6 \text { months, } 2 \text { GP per year. Costs are applied to all patients in the adjuvant imatinib arm } \\
\text { who have not experienced a recurrence. }\end{array}$ \\
\hline Adverse events (cost per event) & $\$ 311$ & $\begin{array}{l}\text { Based on frequency of top } 15 \text { adverse events from the Z9001 clinical study report and unit costs } \\
\text { from the Medicare Physician's Fee and Coding guide. }{ }^{1,24}\end{array}$ \\
\hline Recurrent GIST & $\$ 133$ & Based on 1 GE every 3 months, 1 CT every 3 months, and 2 GP per year (NCCN).14 \\
\hline $\mathrm{BSC}$ & $\$ 4,099$ & $\begin{array}{l}\text { Estimates for the cost of BSC were derived from reported costs per month in the last year of life } \\
(\$ 7,030) \text { and the cost of a continuing phase of cancer }(\$ 2,062) \text { with no active treatment. }{ }^{25} \text { Based } \\
\text { on the proportion of individuals in the model in the last year of life }(41 \%) \text { and those in a continu- } \\
\text { ing phase of cancer }(59 \%) \text { in the first year, we calculated a weighted average cost for BSC. }\end{array}$ \\
\hline One-time cost of recurrence & $\$ 13,214$ & $\begin{array}{l}\text { Assumed that first recurrence is associated with a l-time cost that includes: } 1 \text { GP, } 1 \text { specialist } \\
\text { visit, l CT, and surgical resection; the surgical resection cost was estimated as } \$ 12,753.22\end{array}$ \\
\hline \multicolumn{3}{|c|}{$\begin{array}{l}{ }^{a} \text { Calculated as }(0.59 *[4 * \$ 99+4 * \$ 331+2 * \$ 39]+0.41 *[2 * \$ 99+2 * \$ 331+2 * \$ 39]) / 12 \text {. } \\
{ }^{b} \text { Calculated as }(0.41 * \$ 7,030)+(0.59 * \$ 2,062) \text {. }\end{array}$} \\
\hline \multicolumn{3}{|c|}{$\begin{array}{l}\text { BSC = best supportive care; } C P T=\text { Current Procedural Terminology; } C T=\text { computed tomography scan; ESMO = European Society of Medical Oncology; GE=gastroenterolo- } \\
\text { gist visit; GP=general practitioner visit; GIST = gastrointestinal stromal tumors; } m g=\text { milligrams; } N C C N=\text { National Comprehensive Cancer Network; } Z 9001=\text { American } \\
\text { College of Surgeons Oncology Group clinical trial (DeMatteo et al. } 20091) \text {. }\end{array}$} \\
\hline
\end{tabular}

1-year period, monthly probability of 0.014). ${ }^{1}$ This assumption was conservative because assuming discontinuation for other reasons would reduce the predicted costs associated with adjuvant imatinib treatment.

Post-Recurrence Transition Probabilities and Treatment Pathways. Estimates of discontinuation and progression during treatment for recurrence were obtained from a published study of imatinib in the treatment of advanced or metastatic GIST. ${ }^{8}$ Data for discontinuation and progression, survival on second-line treatment with sunitinib, and the rate of progression on second-line treatment with imatinib $800 \mathrm{mg}$ per day were estimated from published literature. ${ }^{8,17,18}$

Data on specific treatment pathways for patients who experience GIST recurrence following surgical resection were limited. We therefore invited 37 global GIST medical experts to participate in a survey to establish estimates of these parameters. Fourteen of these medical experts from 7 different countries agreed to participate in the survey. We administered the survey as a structured telephone interview and asked physicians about treatment patterns for GIST patients at varying stages of disease and treatment. In this model, the proportions of patients treated with TKI therapy (imatinib or sunitinib) or BSC after failure on imatinib $400 \mathrm{mg}$ per day or imatinib 800 mg per day were chosen to reflect the responses from the 4 participating U.S. GIST medical experts.

Mortality. Patients in the recurrence-free GIST health state 
were assumed to be free of GIST after surgery and therefore not at increased risk of death from GIST. All patients in the model were at risk of age-specific background mortality estimated from U.S. life tables. ${ }^{19}$ Patients with recurrent GIST were assumed to be at increased risk of death, with probabilities estimated from published studies of imatinib and sunitinib, as well as epidemiological studies of GIST. 3,8,17,20

Costs. Costs can be categorized as drug, medical care associated with a health state, or a one-time cost associated with recurrence or adverse events. Costs associated with health states comprise visits, tests, and procedures typical for that health state. All costs in the model reflected 2009 dollars; that is, for unit cost estimates derived from years other than 2009, costs were adjusted to 2009 dollars using the medical care component of the Consumer Price Index. ${ }^{21}$ All visits/procedures were assigned costs from the published literature. ${ }^{22-24}$ The frequencies of visits and tests were estimated from the clinical trial study protocol and other published literature..$^{12,14,25,26}$ For patients in the imatinib arm, costs for monitoring were assumed to continue after completion of the 12 months of active treatment; this assumption was made to be consistent with the clinical trial protocol.

Analyses. Results were calculated as the overall budgetary impact and as per member per month (PMPM) costs in each of the first 3 years after the introduction of adjuvant imatinib. Budgetary impact was calculated by subtracting total expected costs in the current scenario (without adjuvant imatinib) from total expected costs in the future scenario (with adjuvant imatinib). PMPM costs were calculated as the total budgetary impact divided by the plan population, divided by 12 . Results represent the budgetary impact of imatinib as adjuvant therapy only in patients with surgically resected localized Kit-positive GIST, the tenth approved indication for imatinib, and do not consider off-label use of the product.

Sensitivity analyses were performed to test the robustness of model results to changes in key parameters. Sensitivity analyses were performed around the population incidence, where we estimated the budgetary impact at twice the base-case incidence $(0.72$ per 100,000$)$ and one-half the base-case incidence $(0.18$ per 100,000$)$. We also varied cost and efficacy (recurrence probabilities) parameters, discontinuation probabilities, and the proportion treated with each therapy for recurrence by $+/-25 \%$ (high, low), to test the robustness of model results to changes in these parameters.

\section{Results}

\section{Budgetary Impact Analysis}

Table 3 presents the number of patients with localized resected GIST, the number of recurrences avoided, and the costs and cost-offsets associated with adjuvant imatinib in the first 3 years after its introduction. The model predicted an estimated net budgetary impact of $\$ 465,126$ in the first year, $\$ 639,159$ in the second year, and $\$ 833,044$ in the third year after introduction. The PMPM predicted budgetary impact was less than $\$ 0.01$ in the first year after introduction and approximately $\$ 0.01$ in the second and third years.

The additional cost of treatment with adjuvant imatinib (\$526,709 in the first year, $\$ 795,861$ in the second year, and $\$ 1,066,893$ in the third year, inclusive of pharmacy treatment and increased monitoring costs) was partially offset by avoiding and delaying recurrences. The model predicted that in a plan population of 10 million members, with 36 incident cases of localized GIST per year, adjuvant imatinib will avoid or delay 2 recurrences in the first and second years and 3 recurrences in the third year ( 7 total over the 3-year period). Predicted cost offsets attributable to avoided treatment and medical care costs associated with recurrence were $\$ 61,583$ in the first year, $\$ 156,702$ in the second year, and $\$ 233,849$ in the third year, where the average cost of recurrent GIST is approximately $\$ 39,000$ per year.

\section{Sensitivity Analyses}

Disease incidence is a major driver of budgetary impact. As expected, when the incidence was assumed to be twice the rate of the base-case $(0.72$ per 100,000$)$, the overall budgetary impact approximately doubled $(\$ 929,204$ in the first year, $\$ 1.277$ million in the second year, and $\$ 1.664$ million in the third year). Similarly, when the incidence was assumed to be one-half the rate of the base-case $(0.18$ per 100,000$)$, the overall budgetary impact was reduced by about one-half.

The impact of $+/-25 \%$ changes in cost and recurrence parameters on the budgetary impact of adjuvant imatinib 3 years after its introduction are presented in a tornado diagram in Figure 2. The tornado diagram displays the budgetary impact relative to the base-case budgetary impact in the third year after the introduction of adjuvant imatinib $(\$ 833,044)$; the length of the bars indicates the magnitude difference between the expected budgetary impact when the parameter is varied by $25 \%$ of the base-case value. The 10 parameters with the greatest impact on model results are displayed.

The budgetary impact of adjuvant imatinib in the third year after its introduction is most sensitive to changes in the price of imatinib. If the price of imatinib were reduced by $25 \%$, the projected budgetary impact would be $\$ 579,684$ (30\% lower); if the price increased by $25 \%$, the projected impact would be $\$ 1.084$ million (30\% higher). The budgetary impact is also sensitive to rates of recurrence, with the budgetary impact most sensitive to rates of recurrence in the first year for patients treated with surgical resection only ( $+/-11 \%$ change) Changes of $+1-25 \%$ in other rates of recurrence (e.g., the rate of recurrence for adjuvant imatinib) had less impact (approximately $2 \%$ ). All other parameters, including the proportions of patients treated with each of the treatment options for 
TABLE 3 Budgetary Impact Results in the First 3 Years After the Introduction of Imatinib as Adjuvant Therapy for Localized Resected GIST ${ }^{a}$

\begin{tabular}{|c|c|c|c|c|c|c|c|c|c|}
\hline & \multicolumn{3}{|c|}{ Current Scenario } & \multicolumn{3}{|c|}{ Future Scenario } & \multicolumn{3}{|c|}{ Difference [PMPM] } \\
\hline & Year 1 & Year 2 & Year 3 & Year 1 & Year 2 & Year 3 & Year 1 & Year 2 & Year 3 \\
\hline \multicolumn{10}{|l|}{ Number of GIST patients } \\
\hline $\begin{array}{l}\text { Incident cases treated with } \\
\text { surgical resection + adjuvant } \\
\text { imatinib }\end{array}$ & 0 & 0 & 0 & 10.8 & 16.2 & 21.6 & - & - & - \\
\hline $\begin{array}{l}\text { Incident cases treated with } \\
\text { surgical resection only }\end{array}$ & 36 & 36 & 36 & 25.2 & 19.8 & 14.4 & - & - & - \\
\hline Prevalent cases and deaths & 0 & 36 & 72 & 0 & 36 & 72 & - & - & - \\
\hline $\begin{array}{l}\text { Total patients being followed } \\
\text { in model }{ }^{b}\end{array}$ & 36 & 72 & 108 & 36 & 72 & 108 & - & - & - \\
\hline Total number of recurrences & 7 & 9 & 10 & 5 & 7 & 7 & -2 & -2 & -3 \\
\hline \multicolumn{10}{|l|}{ Costs } \\
\hline \multicolumn{10}{|l|}{ Nonrecurrent GIST } \\
\hline Adjuvant imatinib & $\$ 0$ & $\$ 0$ & $\$ 0$ & $\$ 505,144$ & $\$ 757,717$ & $\$ 1,010,289$ & $\$ 505,144$ & $\$ 757,717$ & $\$ 1,010,289$ \\
\hline Medical care/monitoring costs & $\$ 46,824$ & $\$ 86,966$ & $\$ 124,330$ & $\$ 68,388$ & $\$ 125,111$ & $\$ 180,935$ & $\$ 21,564$ & $\$ 38,145$ & $\$ 56,605$ \\
\hline Total & $\$ 46,824$ & $\$ 86,966$ & $\$ 124,330$ & $\$ 573,533$ & $\$ 882,827$ & $\$ 1,191,224$ & $\$ 526,709$ & $\$ 795,861$ & $\$ 1,066,893$ \\
\hline \multicolumn{10}{|l|}{ Recurrent GIST } \\
\hline Pharmacy & $\$ 168,830$ & $\$ 494,002$ & $\$ 772,556$ & $\$ 130,149$ & $\$ 382,845$ & $\$ 606,311$ & $-\$ 38,681$ & $-\$ 111,157$ & $-\$ 166,245$ \\
\hline Medical care costs & $\$ 83,937$ & $\$ 134,435$ & $\$ 148,345$ & $\$ 62,153$ & $\$ 99,951$ & $\$ 112,308$ & $-\$ 21,784$ & $-\$ 34,484$ & $-\$ 36,037$ \\
\hline $\mathrm{BSC}$ & $\$ 4,367$ & $\$ 42,847$ & $\$ 122,594$ & $\$ 3,249$ & $\$ 31,785$ & $\$ 91,027$ & $-\$ 1,118$ & $-\$ 11,062$ & $-\$ 31,567$ \\
\hline Total & $\$ 257,134$ & $\$ 671,284$ & $\$ 1,043,495$ & $\$ 195,551$ & $\$ 514,582$ & $\$ 809,646$ & $-\$ 61,583$ & $-\$ 156,702$ & $-\$ 233,849$ \\
\hline Total Costs & $\$ 303,958$ & $\$ 758,251$ & $\$ 1,167,825$ & $\$ 769,084$ & $\$ 1,397,410$ & $\$ 2,000,869$ & $\begin{array}{l}\$ 465,126 \\
{[<\$ 0.01]}\end{array}$ & $\begin{array}{l}\$ 639,159 \\
{[\$ 0.01]}\end{array}$ & $\begin{array}{l}\$ 833,044 \\
{[\$ 0.01]}\end{array}$ \\
\hline
\end{tabular}

a Difference in total costs between the future and current scenarios is equal to the budgetary impact. Budgetary impact results represent only those related to imatinib as adjuvant therapy for localized resected GIST.

bTotal patients followed in the model each year include incident cases (treated with either surgical resection only or surgical resection plus adjuvant imatinib), prevalent cases (i.e., still living and incident in a previous year), and patients who have died.

$B S C=$ best supportive care; GIST = gastrointestinal stromal tumors; $P M P M=$ per member per month.

recurrence and progression, had minimal impact on model results (less than $2 \%$ change). In addition, increasing the proportion of patients treated with adjuvant imatinib each year increases the budgetary impact directly. The proportion of patients treated with adjuvant imatinib was assumed not to exceed $75 \%$ of patients with localized resected GIST in sensitivity analyses because $75 \%$ was the assumed proportion of patients who are Kit-positive and therefore eligible for adjuvant imatinib. ${ }^{27}$

\section{Discussion}

In a hypothetical health plan of 10 million members, the net budgetary impact of adjuvant imatinib was estimated to be $\$ 465,126$ in the first year, $\$ 639,159$ in the second year, and $\$ 833,044$ in the third year after its introduction. The total impact to health plans was calculated as a function of the number of persons treated, the increased costs associated with the treatment itself, and cost offsets associated with avoiding GIST recurrences. The model predicted that adjuvant imatinib will avoid or delay a total of 7 recurrences over a 3-year period in a hypothetical plan population of 10 million persons, partially offsetting the costs of imatinib treatment. The increase in budgetary impact over the 3 years is due to an increase in utilization of adjuvant imatinib each year. Although the estimated impact increased over the 3 years, the estimated PMPM impact was $\$ 0.01$ in the third year.

\section{Limitations}

First, results of the ACOSOG Z9001 study were used to estimate the probability of recurrence over a 3-year period for patients in both treatment arms. The median follow-up time in the ACOSOG study was 19.7 months, and estimates of recurrence in the third year are based on small sample sizes $(n=105$ for the imatinib arm and $\mathrm{n}=89$ for the surgical resection only arm). Because rates from the ACOSOG trial were used to estimate recurrence for both the adjuvant imatinib arm and the surgical resection only arm, the direction of any potential bias from using these rates is unknown.

Second, we accounted for discontinuation only due to adverse events. This approach is conservative; a greater rate of discontinuation would reduce costs of imatinib but would not affect recurrence probabilities because discontinuation is implicit in the RFS analysis. Additionally, because discontinuation probabilities are based on clinical trial results and we do 
FIGURE 2 Tornado Diagram Presenting Sensitivity Analyses of Budgetary Impact of Adjuvant Imatinib in the Third Year After Introduction to 25\% Changes in Costs and Transition Probabilities

Monthly cost of imatinib adjuvant treatment (low-high)

Rate of GIST recurrence, no treatment, year 1 (high-low) ${ }^{\mathrm{a}}$

Imatinib 400 mg per day drug cost following recurrence (high-low)a

Probability of discontinuing adjuvant imatinib (high-low) ${ }^{\mathrm{a}}$

Imatinib $400 \mathrm{mg}$ per day medical costs, 4 months to 2 years (low-high)

Rate of GIST recurrence, adjuvant imatinib, year 1 (low-high)

Rate of GIST recurrence, no treatment, year 2 (high-low) $^{\mathrm{a}}$

Cost of recurrence-free GIST, first 3 years (high-low) a

Total one-time cost of recurrence (high-low)

Rate of GIST recurrence, adjuvant imatinib, year 3 (low-high)

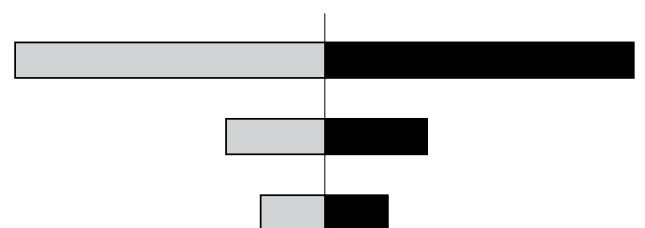

\begin{tabular}{|c|c|c|c|c|c|c|c|c|}
\hline 400 & 500 & 600 & 700 & 800 & 900 & 1000 & 1100 & 1200 \\
\hline
\end{tabular}

andicates that when the value of a parameter is increased (e.g., high) the model produces a result that is lower than the base-case budgetary impact.

not have estimates of discontinuation for other reasons, we did not estimate the effects of other reasons for discontinuation (e.g., out-of-pocket costs).

Third, the model is subject to limitations inherent in the study design. The decision analytic model is a simplified representation of disease natural history and associated outcomes and may not account for all possible health states and complications associated with disease. In addition, the data used to estimate transition probabilities and costs were derived and synthesized from a variety of sources, and this process is subject to bias. We also used the ACOSOG trial clinical study protocol to determine the resource utilization associated with monitoring patients treated with adjuvant imatinib after surgical resection of GIST. Protocol-driven care generally includes more visit and test use than would be observed in a real-world setting, thus making our estimates of the cost of monitoring imatinib patients conservative, that is, potentially higher than they would be in clinical practice. We performed sensitivity analyses to quantify how different parameter assumptions would change results, and we identified that the model was most sensitive to recurrence rates and drug costs.

\section{Conclusion}

A decision analytic model was used to estimate the potential budgetary impact of the introduction of imatinib as adjuvant treatment in accordance with its labeled indication for patients with surgically resected, Kit-positive GIST to health plans in the United States. The model predicted that use of adjuvant imatinib would lead to fewer patients experiencing a cancer recurrence, which is beneficial from a clinical perspective, and to cost offsets associated with avoiding treatment for recurrence. The introduction of adjuvant imatinib would increase a health plan budget by an estimated $\$ 833,000$ in a plan of 10 million members, or approximately $\$ 0.01 \mathrm{PMPM}$, in the third year after introduction.

\section{Authors}

JAIME L. RUBIN, MA, is Project Manager, Health Economics \& Outcomes; DOUGLAS C.A. TAYLOR, MBA, is Director, Health Economics \& Strategic Consulting, Ingenix Fellow; and MYRLENE SANON, MPH, is Health Economics Research Analyst, i3 Innovus, Medford, Massachusetts. JOHN H. COOMBS, PharmD, MBA, is Associate Director, Novartis Pharmaceuticals Corporation, Florham Park, New Jersey; and VAMSI K. BOLLU, PhD, MBA, is Associate Director, Novartis Pharmaceuticals Corporation, East Hanover, New Jersey.

AUTHOR CORRESPONDENCE: Jaime L. Rubin, MA, Health Outcomes, i3 Innovus, 10 Cabot Rd., Ste. 304, Medford, MA 02155. Tel.: 781.518.4032; Fax: 781.338.9522;

E-mail: Jaime.Rubin@i3innovus.com 


\section{DISCLOSURES}

This research was sponsored by Novartis, including payment for researchrelated travel and for development of the manuscript, and 2 of the authors are employees of Novartis

Concept and design were performed by Rubin, Taylor, and Coombs. Rubin, Coombs, and Sanon collected the data. Data interpretation was performed by Sanon and Rubin, with assistance from the other authors. Rubin was primarily responsible for writing the manuscript with input from the other authors. Manuscript revisions were performed primarily by Rubin and Bollu, assisted by Sanon

\section{REFERENCES}

1. DeMatteo RP, Ballman KV, Antonescu CR, et al. Adjuvant imatinib mesylate after resection of localised, primary gastrointestinal stromal tumour: a randomised, double-blind, placebo-controlled trial. Lancet. 2009;373(9669):1097-104. Available at: http://www.ncbi.nlm.nih.gov/pmc/ articles/PMC2915459/pdf/nihms108869.pdf. Accessed August 18, 2010

2. DeMatteo RP, Gold JS, Saran L, et al. Tumor mitotic rate, size, and location independently predict recurrence after resection of primary gastrointestinal stromal tumor (GIST). Cancer. 2008;112(3):608-15. Available at: http://www3.interscience.wiley.com/cgi-bin/fulltext/117861982/PDFSTART Accessed August 13, 2010

3. Tran T, Davila JA, El-Serag HB. The epidemiology of malignant gastrointestinal stromal tumors: an analysis of 1,458 cases from 1992-2000. Am J Gastroenterol. 2005;100(1):162-68.

4. Nilsson B, Bümming P, Meis-Kindblom JM, et al. Gastrointestinal stromal tumors: the incidence, prevalence, clinical course, and prognostication in the preimatinib mesylate era-a population-based study in western Sweden. Cancer. 2005;103(4):821-29. Available at: http://www3.interscience.wiley. com/cgi-bin/fulltext/109865247/PDFSTART. Accessed August 13, 2010.

5. Bümming P, Ahlman H, Andersson J, Meis-Kindblom JM, Kindblom LG, Nilsson B. Population-based study of the diagnosis and treatment of gastrointestinal stromal tumours. Br J Surg. 2006;93(7):836-43.

6. DeMatteo RP. The GIST of targeted cancer therapy: a tumor (gastrointestinal stromal tumor), a mutated gene (c-kit), and a molecular inhibitor (STI571). Ann Surg Oncol. 2002;9(9):831-39.

7. DeMatteo RP, Lewis JJ, Leung D, Mudan SS, Woodruff JM, Brennan MF. Two hundred gastrointestinal stromal tumors: recurrence patterns and prognostic factors for survival. Ann Surg. 2000;231(1):51-58. Available at: http:// www.ncbi.nlm.nih.gov/pmc/articles/PMCl420965/pdf/20000100s00008p51 pdf. Accessed August 13, 2010.

8. Verweij J, Casali PG, Zalcberg J, et al. Progression-free survival in gastrointestinal stromal tumours with high-dose imatinib: randomised trial. Lancet. 2004;364(9440):1127-34.

9. Nilsson B, Sjölund K, Kindblom LG, et al. Adjuvant imatinib treatment improves recurrence-free survival in patients with high-risk gastrointestinal stromal tumours (GIST). Br J Cancer. 2007;96(11):1656-58. Available at: http://www.ncbi.nlm.nih.gov/pmc/articles/PMC2359924/pdf/6603797a.pdf. Accessed August 13, 2010.

10. Gleevec (imatinib mesylate) for oral use. Available at: http://www. pharma.us.novartis.com/product/pi/pdf/gleevec_tabs.pdf. Accessed August 13, 2010 .

11. Dematteo RP, Heinrich MC, El-Rifai WM, Demetri G. Clinical management of gastrointestinal stromal tumors: before and after STI-571. Hum Pathol. 2002;33(5):466-77.

12. Casali PG, Jost L, Reichardt P, Schlemmer M, Blay JY; ESMO Guidelines Writing Group. Gastrointestinal stromal tumors: ESMO clinical recommendations for diagnosis, treatment and follow-up. Ann Oncol. 2008;19(Suppl 2):ii35-ii38
13. U.S. Department of Health and Human Services. U.S. Food and Drug Administration. Imatinib mesylate. February 24, 2010. Available at: http:// www.fda.gov/AboutFDA/CentersOffices/CDER/ucml29210.htm. Accessed August 13, 2010.

14. Demetri GD, Benjamin RS, Blanke CD, et al. NCCN Task Force report: management of patients with gastrointestinal stromal tumor (GIST) update of the NCCN clinical practice guidelines. J Natl Compr Canc Netw. 2007;5(Suppl 2):S1-S29

15. Rubin J, Sanon M, Taylor D, Coombs J, Williams D, Thompson D. Epidemiology of surgically resected localized gastrointestinal stromal tumors (GIST) in the US. Poster presented at: 2009 American Society of Clinical Oncology Gastrointestinal Cancers Symposium; January 15, 2009; San Francisco, CA.

16. Surveillance, Epidemiology, and End Results (SEER) Program (www.seer. cancer.gov) SEER*Stat Database: Incidence - SEER 9 Regs Research Data, Nov 2007 Sub (1973-2005) <Katrina/Rita Population Adjustment> - Linked To County Attributes - Total U.S., 1969-2005 Counties, National Cancer Institute, DCCPS, Surveillance Research Program, Cancer Statistics Branch, released April 2006, based on the November 2005 submission.

17. Demetri GD, van Oosterom AT, Garrett CR, et al. Efficacy and safety of sunitinib in patients with advanced gastrointestinal stromal tumour after failure of imatinib: a randomised controlled trial. Lancet. 2006;368(9544):1329-38.

18. Contreras-Hernández I, Mould-Quevedo JF, Silva A, et al. A pharmacoeconomic analysis of second-line treatment with imatinib or sunitinib in patients with advanced gastrointestinal stromal tumours. $\mathrm{Br} J$ Cancer. 2008;98(11):1762-68. Available at: http://www.ncbi.nlm.nih.gov/pmc/articles/PMC2410103/pdf/6604367a.pdf. Accessed August 13, 2010.

19. Arias E. United States life tables, 2004. National Vital Statistics Reports. 2007;56(9):1-40. Available at: http://www.cdc.gov/nchs/data/nvsr/nvsr56/ nvsr56_09.pdf. Accessed August 13, 2009.

20. Huse DM, von Mehren M, Lenhart G, et al. Cost effectiveness of imatinib mesylate in the treatment of advanced gastrointestinal stromal tumours. Clin Drug Investig. 2007;27(2):85-93.

21. U.S. Department of Labor, Bureau of Labor Statistics. Measuring price change for medical care in the CPI. April 12, 2010. Available at: http://www. bls.gov/cpi/cpifact4.htm. Accessed August 17,2010.

22. DRG Expert: A Comprehensive Guidebook to the DRG Classification System, 24th ed. Salt Lake City, UT: Ingenix; 2009

23. Red Book 2009: Pharmacy's Fundamental Reference, 113th ed. Montvale, NJ: Thomson Healthcare; 2009.

24. Physician's Fee and Coding Guide 2009. Vol. 1. Duluth, GA: MAG Mutual Healthcare Solutions, Inc; 2007.

25. Yabroff KR, Lamont EB, Mariotto A, et al. Cost of care for elderly cancer patients in the United States. J Natl Cancer Inst. 2008;100(9):630-41. Available at: http://jnci.oxfordjournals.org/cgi/reprint/100/9/630. Accessed August 13, 2010.

26. U.S. National Institutes of Health. Imatinib mesylate (Gleevec; STI571) in treating patients with primary gastrointestinal stromal tumor that has been completely removed by surgery. ClinicalTrials.gov identifier: NCT00041197. Available at: http://clinicaltrials.gov/ct2/show/NCT00041197 ?term=STI571\&rank=49. Accessed August 13, 2010.

27. Battochio A, Mohammed S, Winthrop D et al. Detection of c-KIT and PDGFRA gene mutations in gastrointestinal stromal tumors: comparison of DHPLC and DNA sequencing methods using a single population-based cohort. Am J Clin Pathol. 2010;133(1):149-55. 